\title{
Instability of helical tip vortices in rotor wakes
}

\author{
J. N. SØRENSEN
}

Department of Mechanical Engineering, Technical University of Denmark, DK-2800 Kongens Lyngby, Denmark

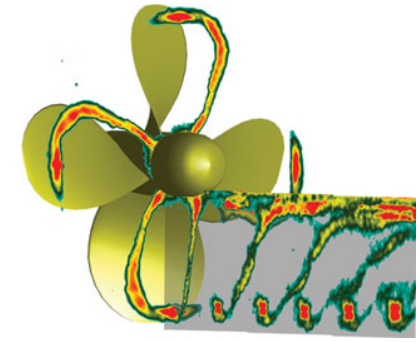

The conditions for the appearance of instabilities in systems of helical vortices constitute an intriguing problem that still remains partly unsolved. The experimental study of Felli, Camussi \& Di Felice (J. Fluid Mech., this issue, vol. 682, 2011, pp. 553) has shed new light on some of the basic mechanisms governing the instability mechanisms.

Key words: aerodynamics, vortex instability, wakes

\section{Introduction}

The flow behind rotor systems, such as helicopters, wind turbines and propellers, consists of a vortex sheet which is formed by the lift distribution along the blades. Due to the interaction between the vortex elements a roll-up process sets in immediately after the vortex sheet is created, which forms strong tip and root vortices in the immediate near-wake behind the rotor. The roll-up process is essentially governed by the interaction law of Biot-Savart. In most cases, the tip/root vortex system is unstable, and due to various instability mechanisms it eventually breaks down and forms small-scale turbulence further downstream. The wake can generally be divided into two distinct parts, near wake and far wake. Near-wake features are related to the genesis of the vortex system where the presence of the rotor is felt directly through the formation of the vortex system. The far wake is usually the downstream position where the wake dynamics no longer depends on specific rotor characteristics and where the flow has broken down and is dominated by small-scale turbulence. The open question, however, is how to establish the relationship between near-wake dynamics and far-wake behaviour. The dynamics of the wake has practical applications for the aerodynamic performance of helicopters (Conlisk 2001) and wind turbines (Vermeer, Sørensen \& Crespo 2003) as well as for ship propellers (Breslin \& Andersen 1994).

The geometry of the wake beneath a helicopter rotor is important for predicting accurately air loads on the blades in hover or vertical flight, and the instability of the wake geometry is felt directly by the rotor and may have an impact on performance, vibrations and noise. Modern wind turbines are often grouped in large parks where the turbines located in the interior of the park are exposed to wake flows from the surrounding turbines. This increases the fatigue loads and hence reduces the lifetime of the turbines. In most cases, the vortices become unstable and break down. It is obvious that if a wind turbine is located in a wake consisting of stable tip 
vortices, the fatigue loading is more severe than if the vortices have broken down by instability. For ship propellers, the destabilization of the tip vortex system may have a large influence on the performance of the rudder and the hull. There are of course major differences between propellers and wind turbine rotors, notably the fact that wind turbines extract energy from the flow and create an expanding wake, whereas propellers add energy to the flow and generate a contracting wake. The stability problem, however, is similar for both types of rotor systems.

\section{Overview}

Inviscid studies by Widnall (1972) indicate the existence of at least three different instability modes in helical vortices. Using single twisted helices, she found that the vortices are unstable to long- and short-wave instabilities, as well as to a mutual inductance mode. The latter occurs when the pitch of the helix decreases and the neighbouring turns of the filament begin to interact strongly. Gupta \& Loewy (1974) were the first to consider the stability of a multiplicity of helical vortices as a means of modelling tip vortices in rotor wakes. They extended the results of Levy \& Forsdyke (1928) and Widnall (1972) by considering the stability of centreline perturbations of the helical vortex system. Later numerical investigations of tip vortex stability beneath a helicopter rotor are due to Bhagwat \& Leishman (2000). In all the mentioned investigations, the helical vortex system was found to be unstable. This result, however, is in contradiction to visualizations of rotor wakes, which show that helical tip vortices subject to small pitch values can be stable (see e.g. Vermeer et al. 2003). A recent theoretical analysis by Okulov \& Sørensen (2007) suggested that it is necessary to consider tip vortices embedded in a wake flow generated by the trailing vortex sheet of the blades in order to include all possible configurations of vortex structures in the stability analysis.

In the paper by Felli, Camussi \& Di Felice (2011), many of these questions were addressed experimentally through comprehensive velocity measurements and highspeed flow visualizations. In the experimental set-up, they used a reference model of a marine propeller and carried out a systematic parameter study in which they changed the advance ratio and the number of blades $(N=2,3$ and 4$)$. From the measurements, it was for the first time possible in detail to follow the onset of instabilities in a system of helical vortices. Traces of all the three instability mechanisms described by Widnall (1972) were indeed detected in the visualizations, although the different instability modes are superimposed, and therefore difficult to discriminate directly from the visualizations. The comparisons with the stability limits determined by the stability model of Okulov (2004) and Okulov \& Sørensen (2007) showed that this model cannot explain all the observed phenomena. In some cases, the vortex system remained stable, although the stability analysis showed the opposite. However, it was not possible to measure the circulation on the rotor blades.

Instead, the strength of the tip vortex was estimated using an additional inviscid numerical simulation. From this, it seems that the circulation of the hub vortex depends on the number of blades and advance ratio, whereas the strength of the tip vortices, in accordance with measured vorticity fields, remains the same regardless of the different propeller configurations. This indicates that it is required with a refined stability analysis in which the contribution of the trailing vortex sheet is also taken into account. Another important observation was that the amplification rate of the mutual-inductance-instability mode (e.g. vortex pairing) increases with increased propeller loading, partly because of a reduction in wake pitch and partly 
(a)

(b)
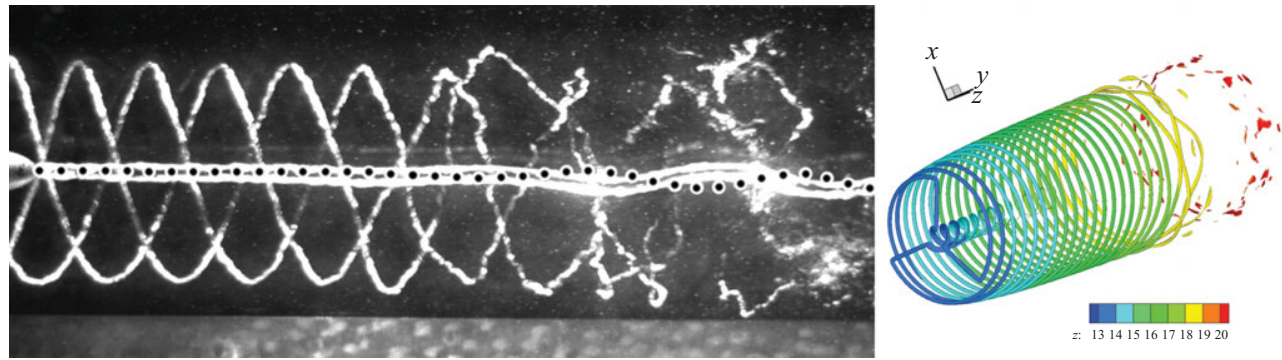

FiguRE 1. Vortex evolution in the wake behind a three-bladed propeller. (a) Experimental visualizations of a marine propeller by Felli et al. (2011). (b) Numerical visualization of the wind turbine rotor by Ivanell et al. (2010). Used with permission.

because of the creation of larger core sizes. Furthermore, it was shown that the mutual-inductance mode was formed through a multi-step grouping mechanism that depended on the number of blades, and that the instability was initiated at the tip vortices through pairing mechanisms that subsequently caused the hub vortex to become unstable. Finally, the hub vortex was found to undergo a double-helix vortex breakdown, with a fundamental frequency corresponding to the frequency of the blade passage $(f=N / T)$.

Many of the results obtained by Felli et al. (2011) are in accordance with recent numerical simulations using the actuator line model developed by Sørensen \& Shen (2002) for studying rotor wakes. In this model, the three-dimensional Navier-Stokes equations are solved with body forces distributed along rotating lines representing the blades of the rotor. The flow field is thus determined by solving the three-dimensional Navier-Stokes equations using large-eddy simulations (LES), whereas the loading on each blade is computed by calculating the local angle of attack to determine the local forces from tabulated aerofoil data. The numerical model formed the basis for a fundamental stability study (Ivanell et al. 2010) in which well-defined disturbances were imposed upstream of a three-bladed rotor to determine the receptivity to various frequencies. In excellent agreement with the measurements of Felli et al. (2011), it was shown that the instability is dispersive and that growth arises for specific frequencies and modes with wavenumbers equal to half-integer multiples of the number of blades, i.e. $N(i-1 / 2)$, where $i=1,2,3, \ldots$, denotes the mode number. Furthermore, the computed results confirmed the experimental observations that the flow is most vulnerable to modes with every other spiral out of phase, and that the mutualinductance instability is associated with vortex pairing. In figure 1 we compare the numerically generated vortex structures with the visualizations from the experiment of Felli et al. (2011). Excellent qualitative agreement is seen to exist between the two visualizations and they both are characterized by containing out-of-phase modes with wavenumbers equal to 4.5 , corresponding to $i=2$.

\section{Future}

The visualizations have elucidated some of the phenomena associated with instabilities of helical vortices in the wake of rotors. However, it is still required to discriminate between the various instability mechanisms and in some cases less 
studied types of instability may be at play. An example of this is the connection between the pairing instability and the appearance of the vortex ring state of a descending helicopter, as discussed by Bolnot, Leweke \& Le Dizès (2011). For wind turbines, the instability and breakdown of the helical vortices only forms the initial part of the wake. Just as important is the connection between the near-wake vortex system and the development of the turbulence in the far wake. In particular, when considering large wind parks, the mutual interaction of the wakes is crucial both with respect to power production and the lifetime of the wind turbines. Thus, there is a need for a better understanding of the relation between the loading of a wind turbine, the subsequent vortex system, the ambient turbulence and the resulting coherent turbulence structures in the wake. As a continuation of the experiment of Felli et al. (2011), it would therefore be of great interest to also study the influence of shear and upstream turbulence on the resulting vortex structures.

\section{References}

Bhagwat, M. J. \& Leishman, J. G. 2000 Stability analysis of helicopter rotor wakes in axial flight. J. Am. Helicopter Assoc. 45, 165-178.

Bolnot, T., Leweke, T. \& Le DizÈs, S. 2011 Spatio-temporal development of the pairing instability in helical vortices. AIAA Paper 2011-3927.

Breslin, J. P. \& Andersen, P. 1994 Hydrodynamics of Ship Propellers. Cambridge University Press.

Conlisk, A. T. 2001 Modern helicopter rotor aerodynamics. Prog. Aerosp. Sci. 37, 419-476.

Felli, M., Camussi, R. \& Di Felice, F. 2011 Mechanisms of evolution of the propeller wake in the transition and far fields. J. Fluid Mech. 682, 5-53.

Gupta, B. P. \& Loewy, R. G. 1974 Theoretical analysis of the aerodynamic stability of multiple, interdigitated helical vortices. AIAA J. 12, 1381-1387.

Ivanell, S., Mikkelsen, R., Sørensen, J. N. \& Henningson, D. 2010 Stability analysis of the tip vortices of a wind turbine. Wind Energy 13, 705-715.

Levy, H. \& Forsdyke, A. G. 1928 The steady motion and stability of a helical vortex. Proc. R. Soc. Lond. A 120, 670-690.

Okulov, V. L. 2004 On the stability on multiple helical vortices. J. Fluid Mech. 521, 319-342.

Okulov, V. L. \& Sørensen, J. N. 2007 Stability of helical tip vortices in a rotor far wake. J. Fluid Mech. 576, 1-25.

Sørensen, J. N. \& Shen, W. Z. 2002 Numerical modeling of wind turbine wakes. J. Fluids Engng 124, 393-399.

Vermeer, L. J., Sørensen, J. N. \& Crespo, A. 2003 Wind turbine wake aerodynamics. Prog. Aerosp. Sci. 39, 467-510.

Widnall, S. E. 1972 The stability of a helical vortex filament. J. Fluid Mech. 54, 641-663. 